\title{
Accelerating OpenVG with multimedia processors on mobile phones
}

\author{
Hwanyong LEE ${ }^{1,2}$, Nakhoon BAEK ${ }^{1,3, a)}$, Inkyun LEE ${ }^{2}$, \\ Jiyoung YOON ${ }^{2}$, Olivier POTHIER ${ }^{4}$, Denis VALLIN ${ }^{5}$, \\ Thierry VAULAY ${ }^{5}$, and Jean-Christophe TROTIN ${ }^{4}$ \\ ${ }^{1}$ School of Computer Sci. and Eng., Kyungpook Nat'l Univ., Daegu, Korea \\ ${ }^{2}$ Huone Inc., Daegu, Korea \\ ${ }^{3}$ Mobile Graphics Inc., Daegu, Korea \\ ${ }^{4}$ ST-Ericsson, Paris, France \\ ${ }^{5}$ ST-Ericsson, Le Mans, France \\ a) Corresponding author.E-mail: oceancru@gmail.com
}

\begin{abstract}
Various handheld devices, in the first place mobile phones, have strong needs regarding 2D vector \& bitmap graphics capabilities, including high performance and quality requirements. OpenVG is one of widely used low-level 2D vector graphics API's. Currently, fully dedicated OpenVG semiconductor chips are relatively expensive and require high power consumption. In contrast, full software implementations show lower performance even with almost $100 \%$ of CPU usage, which would disrupt other concurrent applications. In this paper, we present a new cost-effective way of accelerating OpenVG, based on wide-spread and inexpensive multimedia-processing hardwares, presently on the mobile phones. Through an extensive use of these multimedia processors, we successfully accelerated our AlexVG, an OpenVG softwarebased implementation, especially on its fundamental OpenVG features: bit-block transfer, masking, scissoring, color conversion, image transformation, etc. This accelerated implementation comes with a lower power consumption. It exhibits appropriate performance, reaching more than 20 frames per second, even for complicated graphical user experiences. Its CPU utilization ranges from $20 \%$ to $30 \%$, while the remaining CPU power remains available for other real-time tasks and user applications. This implementation is now commercially available and used in several mid-tier mobile phones.
\end{abstract}

Keywords: OpenVG, mobile phones, acceleration, multimedia hardware

Classification: Electron devices, circuits, and systems

\section{References}

[1] D. Rice and R. J. Simpson, Open VG Specification, version 1.1, Khronos Group, 2008. 
[2] Khronos Group, Khronos group home page, [Online] http://www.khronos.org/

[3] D. Kim, K. Cha, and S. Chae, "A high performance OpenVG accelerator with dual-scanline filing rendering," IEEE Trans. Consum. Electron., vol. 54, no. 3, pp. 1301-1311, 2008.

[4] M. Robart, "OpenVG paint subsystem over OpenGL ES shaders," Digest of Technical Papers International Conference on Consumer Electronics, pp. 1-2, 2009.

[5] H. Lee and N. Baek, "AlexVG: An OpenVG implementation with SVGTiny Support," Computer Standards \& Interfaces, vol. 31, no. 4, pp. 661668, 2009.

[6] Amanith, AmanithVG GLE, [Online] http://www.amanithvg.com/ project.html

[7] Hooked Wireless, [Online] http://www.hookedwireless.com/ OpenVG.html

[8] T. Porter and T. Duff, "Compositing digital images," SIGGRAPH Computer Graphics, vol. 18, no. 3, pp. 253-259, 1984.

[9] STEricsson, [Online] http://www.stericsson.com/platforms/ edge_6517.jsp

[10] STEricsson, [Online] http://www.stericsson.com/platforms/ 6710_HSPA.jsp

\section{Introduction}

Vector graphics features exhibit many advantages including scalability in terms of display size, small file size, lossless compression capability, no compression artifacts, etc. Currently, the need for such features is high and increasing, especially on handheld devices, where appealing user interfaces are becoming must-have's. Although vector graphics features are available as various drawing API's and file formats including Flash, SVG, Cairo, Silverlight, PDF, PostScript and so on, OpenVG [1] from Khronos Group [2] is one of the most suitable API's on the handheld devices.

OpenVG is a royalty-free, cross-platform API that provides a low-level hardware acceleration interface for various vector graphics libraries and applications. OpenVG primarily targets handheld devices that require portable acceleration of high-quality vector graphics for user interfaces and text on small screen devices. Nowadays, there are a few fully-dedicated hardware solutions $[3,4]$ as well as full software implementations [5]. OpenVG semiconductor chips are relatively expensive for ordinary consumer markets while software solutions need to be accelerated.

In this paper, we present a way of accelerating a software OpenVG implementation, named AlexVG [5], with the existing multimedia processing hardware which is widely adopted for music and/or video processing on a variety of handheld devices. Our major goal is to enable OpenVG to provide high performance graphical user experiences with as low CPU utilization as possible, without additional hardware or re-design of hardware, allowing other applications to be concurrently executed on the CPU. 
Though there have been a few trials to accelerate OpenVG with hardware processors, they used fully-dedicated OpenVG or OpenGL ES chips, which are too expensive for being widely used in the consumer market $[6,7]$. Since our work aimed to use inexpensive lower-level multimedia chips, it is distinguishable from the previous ones. Additionally, based on its modular design, our implementation can work with various kinds of low-level chips, including DSP vector processors, bitmap processors, camera processing chips, and others. At least to our knowledge, this paper is the first literature communication dealing with direct hardware acceleration of OpenVG.

\section{Design and implementation}

A theoretical implementation of OpenVG implies an overall pipeline with 8 stages, as described in the OpenVG official specification [1] and also shown in Fig. 1.

OpenVG features suitable for acceleration by the multimedia processor are indicated with the reddish backgrounds in Fig. 1 and can be classified as follows:

- Double Buffering: When the frame buffer memory provides double buffering capability, we can accelerate image handling portions of the

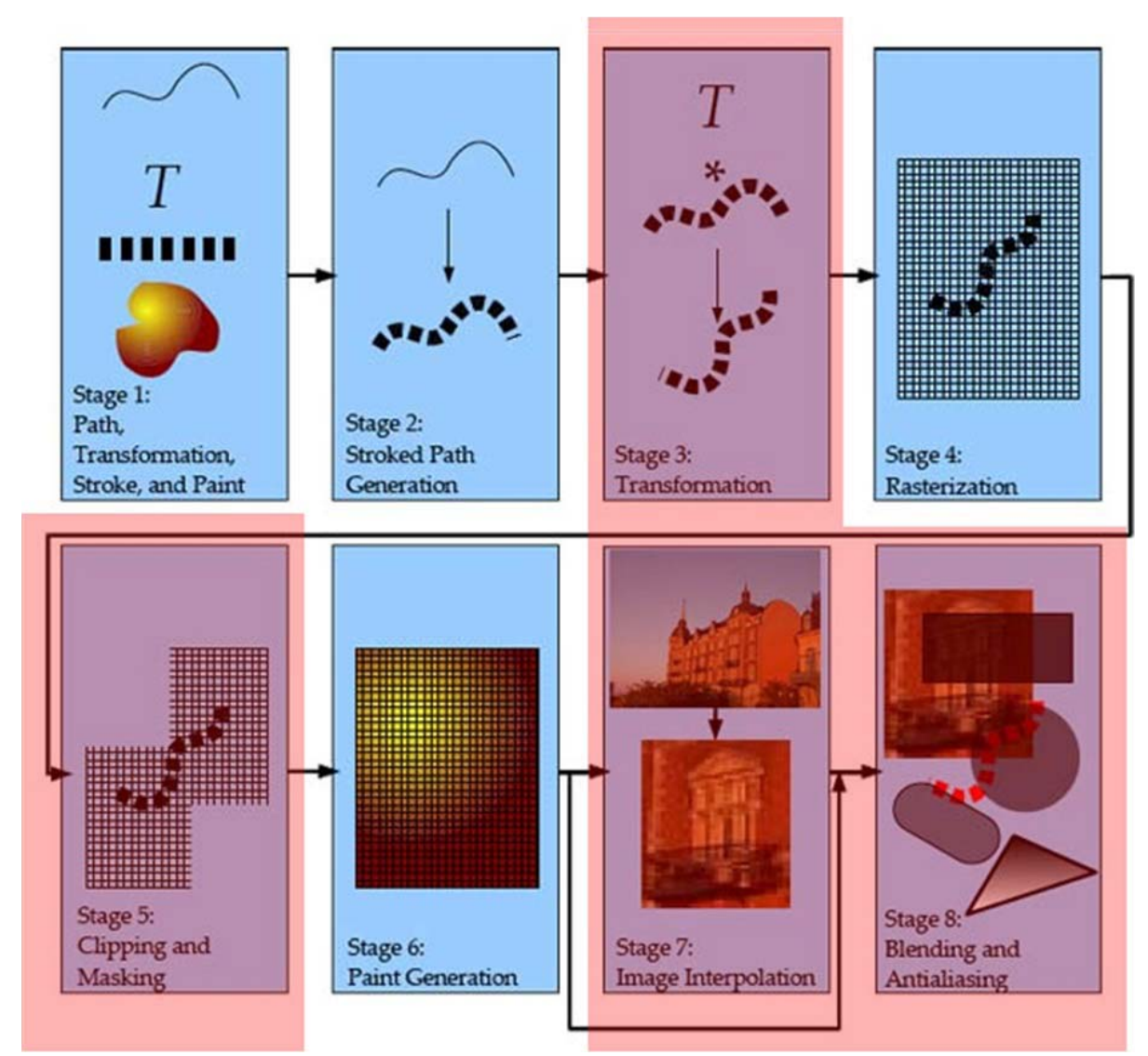

Fig. 1. OpenVG pipeline. Stages marked with reddish backgrounds can be accelerated with the multimedia processors. 
OpenVG pipeline, such as stages 5, 7 and 8. Additional accelerations can be accomplished when partial updates are also available.

- Blending: At its stage 8, the OpenVG pipeline provides blending and antialiasing features. The blending features include general Porter-Duff blending $[8]$ and additional ones like "darken", "lighten" and "additive" operations. These blending features can be accelerated by most multimedia processors.

- Image Copy: If the underlying image buffer provides accelerated block copy or bit-block transfer operations, we can dramatically accelerate OpenVG image handling functions including vgPaintPattern $(\cdots)$, $\operatorname{vgClear}(\cdots)$, vgDrawImage $(\cdots)$ and $\operatorname{vgCopyImage}(\cdots)$.

- Masking and Scissoring: For some multimedia processors supporting alpha masking images, we can improve most of the pixel-level masking, scissoring and blending operations, to finally accelerate imagebased input/output and animation.

- Color Conversion and Color Transformation: Some recent multimedia processors can transform sRGB color values to its corresponding lRGB values and vice versa. We can also accelerate $\operatorname{vgLookup}(\cdots)$ function using RGBA lookup table features.

- Image Transformation: Most multimedia processors support accelerated $3 \times 3$ or $3 \times 2$ matrix multiplications, which can be directly used for the OpenVG image transformation.

- General Arithmetic Calculations: Most arithmetic operations and matrix operations on the 32-bit fixed number representations can be accelerated using multimedia processing chips and even their vector processing features.

After carefully analyzing the above features, we isolated the corresponding code areas from our software implementation [5]. We finally reconstructed the original software implementation into several modules, each of which can be compiled to use the exiting full software implementation or the new multimedia processor accelerated implementation. The final implementation provides the most suitable configurability depending on the available features of the underlying hardware chips.

\section{Implementation results}

Fig. 2 shows our prototype implementation during its development phase. A daughter board with a multimedia processor is attached to the previous software implementation. At this time, the final implementation is now commercially available for various existing multimedia processors, with accelerated graphical user interfaces, as also shown in Fig. 2. For several hardware configurations, we used a set of test programs shown in Fig. 2.(c)-(f) to measure their performance. 

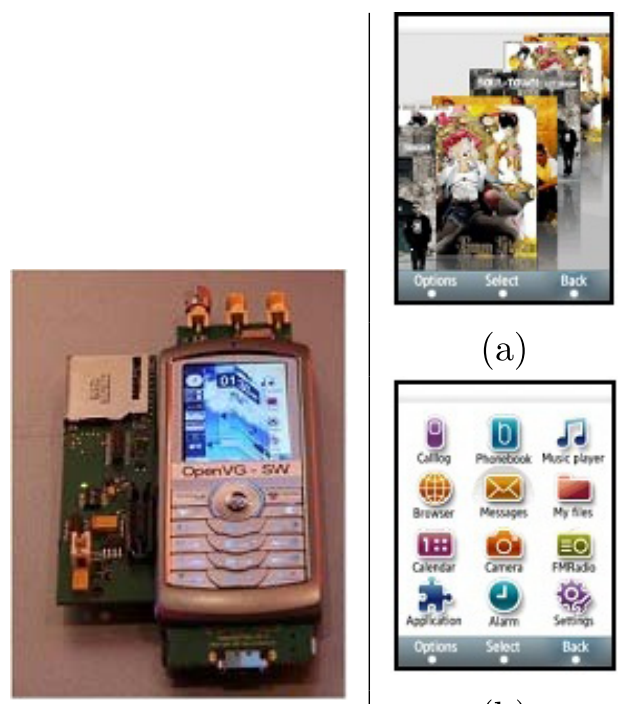

(a)

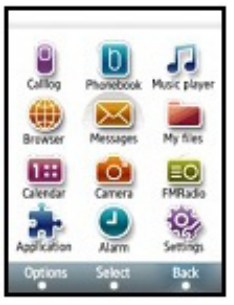

(b)

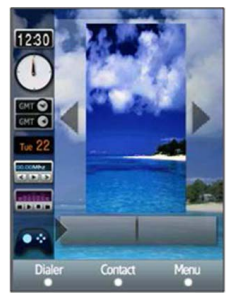

(c) translation

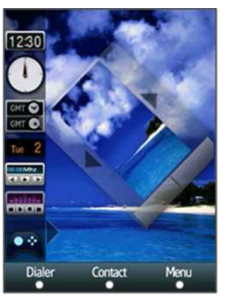

(e) rotation

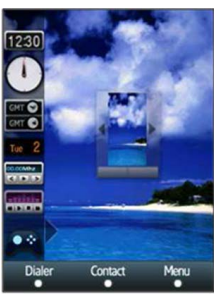

(d) scaling

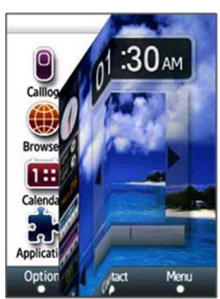

(f) paging

Fig. 2. Left: Our prototype implementation. Middle: Selected GUI implementations accelerated by it. Right: Benchmark programs used in Table I.

Table I. Acceleration measures with some benchmark programs.

\begin{tabular}{|c|c|c|c|c|c|}
\hline \multirow{3}{*}{$\begin{array}{l}\text { test } \\
\text { name }\end{array}$} & \multicolumn{5}{|c|}{$182 \mathrm{MHz}$ CPU with $320 \times 240$ display } \\
\hline & \multicolumn{2}{|c|}{ full s/w sol. } & \multicolumn{2}{|c|}{ multimedia $\mathrm{h} / \mathrm{w}$ sol. } & \multirow{2}{*}{$\begin{array}{c}\text { average } \\
\text { acceleration }\end{array}$} \\
\hline & CPU load & fps & CPU load & fps & \\
\hline translation & $100 \%$ & 4.6 & $20 \%$ & $21.0-24.0$ & $489.13 \%$ \\
\hline scaling & $100 \%$ & $3.0-10.0$ & $20 \%$ & $21.0-24.0$ & $346.15 \%$ \\
\hline rotation & $100 \%$ & 4.5 & $20 \%$ & $18.0-21.0$ & $433.33 \%$ \\
\hline paging & $100 \%$ & $2.6-4.0$ & $20 \%$ & $22.0-33.0$ & $833.33 \%$ \\
\hline & \multicolumn{5}{|c|}{$312 \mathrm{MHz}$ CPU with $320 \times 240$ display } \\
\hline test & \multicolumn{2}{|c|}{ full s/w sol. } & \multicolumn{2}{|c|}{ multimedia $\mathrm{h} / \mathrm{w}$ sol. } & average \\
\hline name & CPU load & fps & CPU load & fps & acceleration \\
\hline translation & $100 \%$ & $5.0-17.0$ & $26 \%$ & $66.0-71.0$ & $622.73 \%$ \\
\hline scaling & $100 \%$ & $5.0-17.0$ & $26 \%$ & $58.0-83.0$ & $640.91 \%$ \\
\hline rotation & $100 \%$ & $7.5-12.0$ & $32 \%$ & $55.0-66.0$ & $620.51 \%$ \\
\hline paging & $100 \%$ & $4.5-6.5$ & $23 \%$ & $125.0-200.0$ & $2954.55 \%$ \\
\hline & \multicolumn{5}{|c|}{$312 \mathrm{MHz}$ CPU with $400 \times 240$ display } \\
\hline test & \multicolumn{2}{|c|}{ full s/w sol. } & \multicolumn{2}{|c|}{ multimedia $\mathrm{h} / \mathrm{w}$ sol. } & average \\
\hline name & CPU load & fps & CPU load & fps & acceleration \\
\hline translation & $100 \%$ & $3.7-12.6$ & $26 \%$ & $49.0-52.0$ & $619.63 \%$ \\
\hline scaling & $100 \%$ & $3.7-12.6$ & $26 \%$ & $43.0-51.0$ & $576.69 \%$ \\
\hline rotation & $100 \%$ & $5.6-8.9$ & $32 \%$ & $43.0-61.0$ & $717.24 \%$ \\
\hline paging & $100 \%$ & $3.7-12.6$ & $23 \%$ & $92.0-148.0$ & $1472.39 \%$ \\
\hline
\end{tabular}

We selected STEricsson's multimedia acceleration hardware [9, 10] and its 2D graphics API for the sample implementation and its benchmark test. This multimedia processing system provides most of the acceleration features

(c) IEICE 2010

DOI: 10.1587/elex.7.1493 Received August 27, 2010

Accepted September 09, 2010

Published October 10, 2010 
listed in Section 2, except a few image blending features.

Table I shows the graphical performances and their comparison with respect to the previous software implementation. User experience appears unsatisfactory when the refresh rate is less than 15 frames per second. Due to the larger screen sizes of nowaday mobile phones, our original software implementation could barely achieve this frame rate. Our multimedia processor accelerated implementation shows dramatic performance improvement.

Note that the $100 \%$ CPU utilization for the full software implementation was lowered to $20 \%$ to $30 \%$, which enhances the real-time multi-processing capabilities. Though most stages in the OpenVG pipeline would be accelerated by multimedia processors, most stroke-related and path generation operations are basically serialzied operations. We assign these serialized operations to CPU, and make balances between the CPU and multimedia processor work loads.

Lower power consumption is another benefit of our implementation. For the configuration of a $312 \mathrm{MHz}$ CPU with the $320 \times 240$ display, as an example, it consumes $120 \mathrm{~mA}$ at its idle state. Our new implementation needs additional $36 \mathrm{~mA}$ for graphical user experience programs, while the previous software implementation $49 \mathrm{~mA}$. It is about $26 \%$ gains in power consumption.

\section{Conclusion}

We analyzed OpenVG features suitable for acceleration with multimedia processors, and finally accomplished hardware-adaptive OpenVG solutions. Our final implementation shows that the OpenVG acceleration on the existing multimedia hardware is cost-effective and achieves relatively high performance, together with lower power consumption.

Our implementation is now commercially serving several mid-tier mobile phones. We also expect that our OpenVG acceleration would be suitable for effectively providing vector graphics features in consumer electronics markets including, not only mobile phones, but also handheld devices and IPTV's.

\section{Acknowledgments}

This research was supported by Basic Science Research Program through the National Research Foundation of Korea (NRF) funded by the Ministry of Education, Science and Technology (Grant 2009-0088544). 